\title{
An Assessment of Design of Observation Network over the Tibetan Plateau Based on Observing System Simulation Experiments (OSSE)
}

\author{
Yueqing LI, Lian YU \\ Institute of Plateau Meteorology, CMA, Chengdu, China \\ and \\ Baode CHEN
}

Shanghai Typhoon Institute, CMA, Shanghai, China

(Manuscript received 16 January 2014, in final form 29 January 2015)

\begin{abstract}
An Observing System Simulation Experiment (OSSE) was conducted to test the rationality and regional representation of the layout of the upper-air observation system over the Tibetan Plateau and neighboring areas and to consider the actual significance of observation stations. The simulations were validated primarily by comparing results from corresponding experiments wherein simulated observations were assimilated into the initial fields or not. The results showed that there were significant differences in prediction for the different layouts. The layout with both existing and planned observation stations was better than the ones with only existing or only planned stations in terms of forecast accuracy, especially around the areas with new observations added. Specifically, for winds, there were large improvements in Xinjiang, Qinghai, Gansu Corridor, Tibet, Yunnan and mid-west and northeast Sichuan. For temperature and relative humidity, the most significant improvement was in Tibet, southwest and east Qinghai, mid-east Sichuan, and mid-north Yunnan. For heights, the major improvements were in Xinjiang, Qinghai, east Tibet, mid-west Sichuan and Yunnan. The results also validated the layout of the observations and indicated the necessity to add planned observations over the Tibetan Plateau and neighboring areas.
\end{abstract}

Keywords Tibetan Plateau; data assimilation; regional climate model; layout of the observation station

\section{Introduction}

The main portion of the Tibetan Plateau is at an altitude above $4500 \mathrm{~m}$, which reaches the middle of the troposphere and is sometimes referred to as the "third pole" of the world. The Tibetan Plateau has significant dynamic and thermodynamic impacts on the regional and global weather and climate and

Corresponding author: Yueqing Li, Institute of Plateau

Meteorology, China Meteorological Administration (CMA),

Chengdu 610072, China

E-mail: yueqingli@163.com

(C)2015, Meteorological Society of Japan they have been intensively investigated by many scholars through field experiments, data and theoretical analyses (Charney 1949; Flohn 1968; Ye and Gao 1979; Yanai et al. 1992; Yanai and Li 1994; Liu and Yin 1999; Liu 2002; Wu et al. 2004). Particularly, there were two large-scale field experiments in the Tibetan Plateau conducted by Chinese scientists. The first Qinghai-Xizang (Tibet) Plateau Meteorological Experiment was conducted in 1979 and gathered very valuable observational data of radiation and heat balance, which were keys for us to come to systematically understand the impact of Tibetan Plateau on the planetary-scale circulation as well on the devel- 
opment of local weather systems, etc. (Yu and Wang 2005; CAMS JICA Implementation Office 2007; Staff members 1984). The Second Tibetan Plateau Atmospheric Experiment (TIPEX) took place in 1998, and the land-atmosphere interaction, the structure of the atmospheric boundary layer, and the cloud and radiation processes in the Tibetan Plateau were further revealed (Tao et al. 1998; Xu et al. 2002, 2003). Moreover, joined by Japan, the U.S. and South Korea, China has conducted various relatively small field experiments and related scientific research, For example, during the period 2004-2009, the JICA and CAMS built a quasi-operational integrated observing system in the Tibetan Plateau including observation system management, data quality control and data processing. The data collected revealed the upstream key region and "strong signal" of disaster weather system, and the system further enhanced the local comprehensive observation and early warning capacity (Yu and Wang 2005; CAMS JICA Implementation Office 2007). The most recent studies indicate that the development of Tibetan vortex has an important impact on the downstream weather based on analyses of potential vorticity and diabatic heating (Zhang et al. 2013; Wu et al. 2013).

Although there have been many studies and field experiments in the Tibetan Plateau, because of poor environmental conditions, limited observation capabilities and steep and complicated terrain as well, the detailed spatial and temporal variations of the atmosphere and surface conditions were still poorly understood in the region. Most critically, the geographic distribution of the regular and conventional observation stations in the Tibetan Plateau was largely determined by district divisions for administrative and governmental convenience, irrespective of considering the scientific rationale and representativeness of observations to be obtained. Therefore, it is imperative and urgent to renovate current observation network in the Tibetan Plateau, however, to build and maintain a new observation system is extremely expensive and unrealistic. The most practical and achievable approach is to integrate new instruments into the existing system to establish a systematic and reliable means of gathering information with sound scientific rationale. Therefore, the potential impact of new design and instruments has to be first determined, and then a proper implementation and operation strategy has to be put forward to maximum favorable impact of the new instruments. In this regard, the Observing System Simulation Experiments (OSSEs) can be used to help guide development of new instru- ments and observation network design in the Tibetan Plateau as OSSEs may be performed during the planning stages.

The OSSE is an efficient way to test and design observing systems (Amold et al. 1986; Masutani et al. 2006). OSSEs are designed to mimic the process of data assimilation. In applications of an atmospheric data assimilation system with regard to real observations, real imperfect observations with errors are taken from the real atmosphere to produce estimates of atmospheric states at sequences of time. In an atmospheric OSSE, simulated observations with simulated errors are obtained from a simulated atmosphere (termed as "nature run" or "NR") and provided to a data assimilation system to produce estimates of those NR states. Since the OSSE deals entirely with simulations, it is not restricted to using only observations that actually exist. Moreover, the underlying "true" atmospheric state is known precisely. These two properties of the OSSE facilitate many types of informative experiments. Masutani et al. (2003) and Masutani et al. (2006) assessed the potential effects of Doppler lidar observed wind data by using OSSE and found that adding high-density wind data could improve the simulations. The further study by Masutani et al. (2010) demonstrated that carefully constructed OSSEs can provide useful information that influences the design of future observing systems. For a Meiyu case in 2003, Chen et al. (2011) added radar, radiosonde, wind profiler, and ground stations data to the OSSE, and found that certain amount of new observational data could improve the short-term precipitation forecast; however, more observations would not necessarily lead to a more accurate forecast.

With economy development in China, the prevention of natural disasters related to weather/climate and the management of their effects have become issues of great concern to the country such that more accurate weather and climate prediction service is called for. Because high-quality observations are essential to produce highly skillful forecasts for the local and downstream areas, there has been a growing demand to innovate present observation network in the Tibetan Plateau and its neighboring area. Prior to deployment of new observation instruments, in this study, we will apply OSSEs to assess the scientific rationality and regional representation of the planned layout of the observation system over the Tibetan Plateau and its neighboring areas and identify observation need through sensitive experiments with synthetic observations. The methodology and data used are briefly described in Section 2. Analysis of numerical experi- 
ments is given in Section 3. Section 4 presents preliminary recommendations for innovating observation system over the Tibetan Plateau. Finally, summary and discussion are addressed in Section 5.

\section{Methods and data}

\subsection{OSSE setup and Data}

An OSSE deals entirely with simulations, it is not restricted to use observations that actually exist or not. We extracted wind and temperature profiles, as the "observations", from analyses of a data assimilation system which can be treated as a Nature Run (NR), then put them into to another analysis system to obtain initial fields for model integrations. The role of "observations" can be examined by comparing the differences between the "NR" and model integration results with/without "observations".

In our study, the high-resolution analysis $\left(0.25^{\circ}\right.$ by $0.25^{\circ}$ ) from the European Centre for Medium-range Weather Forecasts (ECMWF; in short from, EC) is utilized as the nature run; The Weather Research and Forecast model version 3.1 (WRF3.1) is adopted as our primary means to simulate the impacts of new "observations"; Considering the "observations" in the study being "direct" (i.e., no observational operator needed), such as wind or temperature etc., and simplicity as well, the objective analysis (OBSGRID) package provided along with the WRF software is used to incorporate information from "observations" into model's initial fields which were produced from analysis of the NCEP's (National Center of Environment Prediction) Global Forecasting System (GFS). Moreover, to test sensitivity of assimilation methodology, a regional version (modified to be fit to the WRF model) of the Gridpoint Statistical Interpolation (GSI), which was based on three-dimensional variational analysis (3D-VAR), is used.

The locations of "observations" extracted from the "NR" are referred to the layouts of the existing and planned stations (Figs. 1a, b). The OLD was the layout distribution of existing observation station (Fig. 1a); the NEW was the layout distribution of planned observation station (Fig. 1b).

\subsection{Numerical experiments design}

At present, 30 radiosonde stations are scattered in the Tibetan Plateau and its neighboring area, and their distribution comprises the layout of existing observation stations (Fig. 1a). It has been proposed and planned to add the operation of 20 more Radiosondes in the stations wherein only surface observation is currently conducted and the distribution of these planned stations is shown in Figure 1b. It is obvious that for the NEW layout, the 20 stations are still within sensitive and key areas to weather systems but its capacity will be enhanced with more radiosonde observations being added (Wang et al. 2009; Chen et al. 2011; Shen et al. 2008). In fact, Tibetan Plateau and the surrounding area is one of the active centers of weather systems in the northern hemisphere, and Tibetan Plateau vortex (TPV), Southwest China vortex (SWCV) are the major weather systems in these areas. These weather systems always cause rainstorm and flood disasters over the Tibetan Plateau and its downstream regions. The research shows that the activity of TPV is located in the $30-35^{\circ} \mathrm{N}$ belts over the Plateau. It has four generating sources, Naqu over the middle Plateau, Dege over the eastern Plateau, Songpan at the east side of the Plateau, and Shenzha-Gaize over the western Plateau. TPVs, which remove out of Plateau, also has four generating sources, Naqu, Qumalai over the northern Plateau, Dege and Maqin over the northeastern Plateau (Wang et al. 2009). And SWCV has four generating sources, Jiulong over the southeastern Plateau, northwestern of Sichuan basin, southwestern of Sichuan basin, central and northeast of Sichuan basin. SWCV which remove out also has four sources, it consist with the generating sources of SWCV (Shen et al. 2008). Furthermore, the heat of Tibetan Plateau and its surrounding areas, especially the southeastern Tibetan Plateau, main Tibetan Plateau, southern and northeastern Tibetan Plateau, has an important impacts on the evolution of weather systems such as TPV and SWCV, and its precipitation (Wang et al. 1987; Yu 2002; Lv et al. 2006; Zhong et al. 2009; Yang et al. 2010; Wang et al. 2011).

Our primary objective is to evaluate the planned layout of the observation system, to verify the sensitive and key areas over the Tibetan Plateau and its neighboring areas, and to determine the regional representation and actual importance of observation stations. Taking into account the layout of the existing observations in the Tibetan Plateau and its nearby areas, as well as its geographic characteristics, we designed three numerical experiments with different stations distributions:

1. OLD run: the layout of only existing observation stations;

2. NEW run: the layout of only planned observation stations;

3. OLD-NEW run: the layout of existing and planned observation stations joined together.

Three rainfall cases were selected, namely, C10 

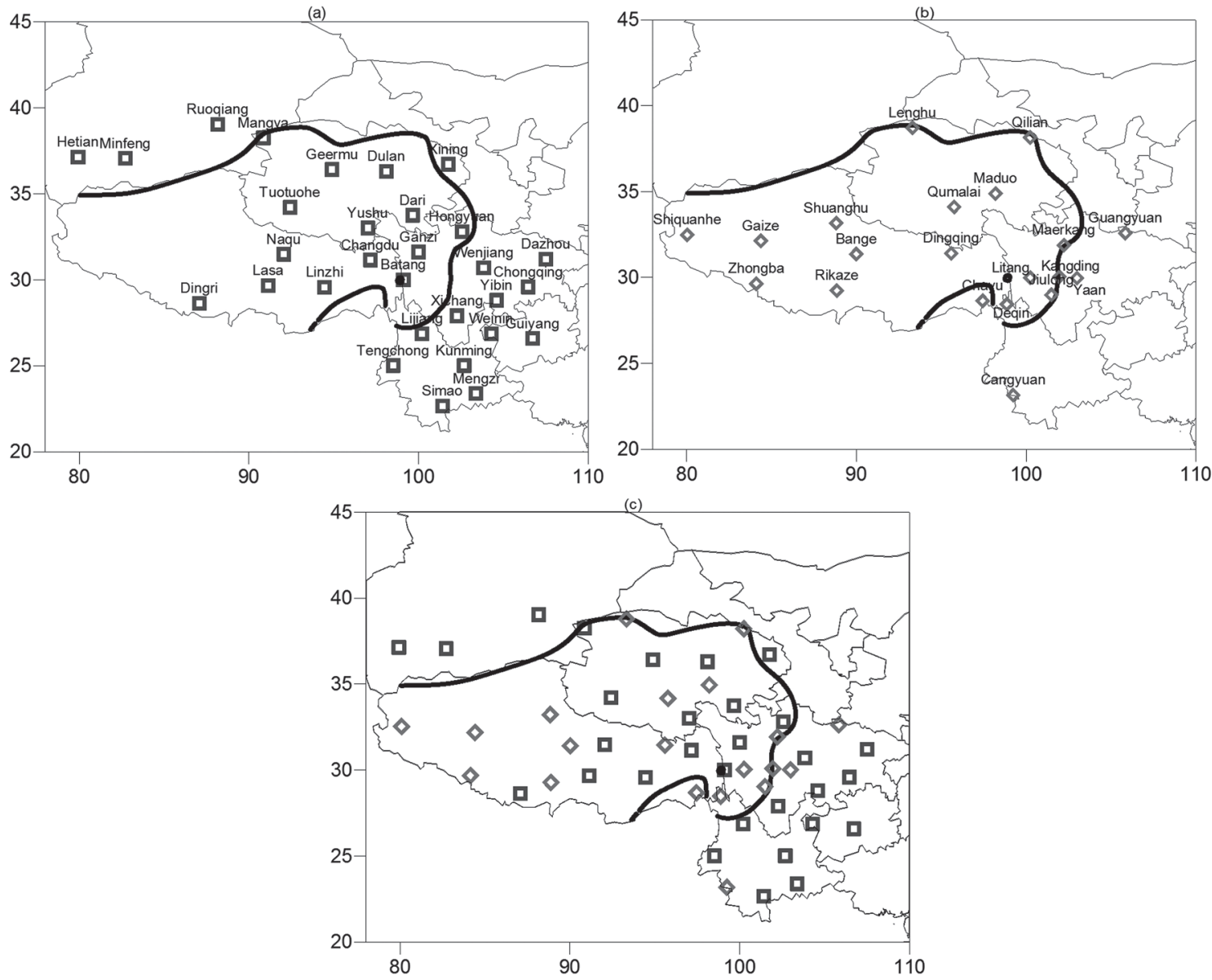

Fig. 1. The (a) OLD, (b) NEW and (c) OLDNEW layout distribution over the Tibetan Plateau and its nearby area. The solid black line represents $2700 \mathrm{~m}$ altitude. The OLD was the layout distribution of existing observation station and the NEW was the layout distribution of planned observation station.

from June 15 to 18 of $2010, \mathrm{C} 11$ from June 1 to 4 of 2011, and C12 from June 25 to 28 of 2012, during which precipitation occurred across whole China with torrential and heavy rainfall in the Tibetan Plateau and its eastern surroundings. A single domain with the center at $\left(105^{\circ} \mathrm{E}, 34^{\circ} \mathrm{N}\right)$ is used, and the horizontal resolution and vertical levels are 15 and $38 \mathrm{~km}$, respectively. The control run (in short from, CNTL) is performed without any "observations" incorporated. The "observations" profiles from the "NR" include zonal wind, meridional wind, temperature, relative humidity, and geo-potential height. Objective analysis (OBSGRID) is used for all three cases and 3D variation assimilation (GSI) just for $\mathrm{C} 10$.

\section{OSSE results}

To examine the effects of the OLD, NEW, and
OLDNEW layout, we calculated the root square mean error (RSME) between the OLD, NEW, and OLDNEW runs and control run (hereinafter referred as to RC), respectively. The control run is performed without any "observation" information assimilated. The RSMEs between the nature run (ECMWF analysis) and the runs with observation added were also computed (hereinafter referred as to RE). Large RC generally indicates high sensitivity of new "observations" to the forecast and small RE suggests low forecast errors; therefore, large RC together with small $\mathrm{RE}$ indicates a positive impact from new "observation". Furthermore, it can be inferentially concluded that the larger difference between $\mathrm{RC}$ and $\mathrm{RE}$ (i.e., $\mathrm{RC}-\mathrm{RE}$ ) is, the more positive impact comes from the "observations". Therefore, in the paper, we will mainly examine the RC - RE distributions, and in 
view of the higher elevations of the Tibetan Plateau, the $500 \mathrm{hPa}$ isobaric surface is selected to compare the regional distribution of the numerical experiment results of different layout tests.

\subsection{Initial profiles}

Figures 2a-1 show the initial RMSE profiles of zonal wind, meridional wind, relative humidity, and temperature for $\mathrm{C} 10$, respectively, which were averaged over the domain for different layouts. In general, at initial time, the RMSE of the control run is the largest, which is reasonable because of no any information from the NR assimilated. The RMSE from the objective analysis method (OBJ) is relatively smaller almost for all variables than those of the control run showing a "locality" characteristic of the OBJ approach wherein observation information was simply inserted into the background fields according to a given influence radius. Unlike the OBJ analysis, the 3D-VAR tends to let observation information spread all of analysis domain and usually certain dynamic constrains (e.g., geostrophic balance) are imposed. The RMSE from 3D-VAR varies greatly for different variables. The vertical distributions of the RMSE from 3D-VAR are quite different to those from OBJ approach and control runs. For the OLDNEW layout, it can be found that the RMSEs of zonal wind and temperature are significantly smaller except near the surface and the RMSE of meridional wind and relative humidity are slightly smaller than the other two RMSE.

For the zonal wind, temperature, and relative humidity of C11 (Figs. 3a, c, d), the RMSEs of OLD, NEW, and OLDNEW layout are significantly smaller than those from CNTL with the smallest RMSE from OLDNEW. The meridional wind (Fig. 3b) RMSEs differences are not large among all runs, it should be pointed out that, for this particular case, at $150 \mathrm{hPa}$ the RMSE of CNTL is smallest. For the zonal wind, meridional wind, temperature, and relative humidity of $\mathrm{C} 12$ (Figs. 4a-d), the RMSE of CTNL is the largest and the RMSE of OLDNEW and OLD are relatively small.

\subsection{Regional distribution of the $R C-R E$}

Figure 5 shows the RC - RE of OLD, NEW, and OLDNEW tests in 2010 with 3D-VAR approach. For the zonal wind (Figs. 5a-c), there are apparent differences between the forecast results in the OLD, NEW, and OLDNEW tests. In the OLD and NEW test forecast results, the improvements of zonal wind (large $\mathrm{RC}-\mathrm{RE}$ ) are mainly distributed in near the layout site and its surrounding area. The improved areas in the OLD layout test are mainly distributed in southeast Xinjiang, the Tibetan Plateau, central and south of Qinghai, central and western of Sichuan and Yunnan. The improved areas in the NEW layout test are mainly distributed in southern Xinjiang, eastern Tibetan Plateau, northern Qinghai, the Hexi Corridor and western Sichuan plateau. In the OLDNEW layout test forecast results, the improved areas are combined with the OLD and NEW layout test and mainly distributed in three regions. The first improved areas in the OLDNEW layout test are mainly distributed in the northern Tibetan Plateau, southern Xinjiang, northern Qinghai, Hexi Corridor and Qilian Mountains. In these regions, although there are only seven layout points, there was a very significant and widespread improvement, especially in the eastern Tarim Basin and the Hexi Corridor. While the results show that the forecast results are very sensitive to layout points "observations" in these areas, the layout points "observations" have a significant impact for forecast results and have a good regional representation. The second areas of improvement in the OLDNEW layout test are mainly distributed in the main area of the Tibetan Plateau, including the whole of Tibet, Qinghai, the central and western of Sichuan. In these regions, there are 31 layout points. There are 13 layout points in Tibet that are mainly distributed in the central and southern portions of the Tibetan Plateau. The improvement is apparent, particularly in Ali, Naqu, Rikaze, Linzhi, and Changdu in Tibet, areas that are also important sources of plateau vortex and heat source sensitive areas. However, the west Kunlun Mountains and the Kekexili almost have no improvement. There are seven layout points in Qinghai that are mainly distributed in the central and southern regions of Qinghai where there are sensitive areas of plateau vortex and heat source. For an obvious improvement of these areas, but the layout points are relatively sparse, the improvement of forecast results is mainly concentrated in a small area within the layout point and its surroundings. There is little improvement in the east Kunlun, east Qinghai, Dege, and Maqin where is near the border between Qinghai and Sichuan. While the Derge, Maqin, and Qumalai are heat source sensitive areas and an important source of plateau vortex. There are 11 layout points in Sichuan, and the forecast results improved very significantly for more intensive layouts, especially in Jiulong. Jiulong is an important and sensitive SWCV source and is more sensitive to the synoptic scale systems. The addition of the 

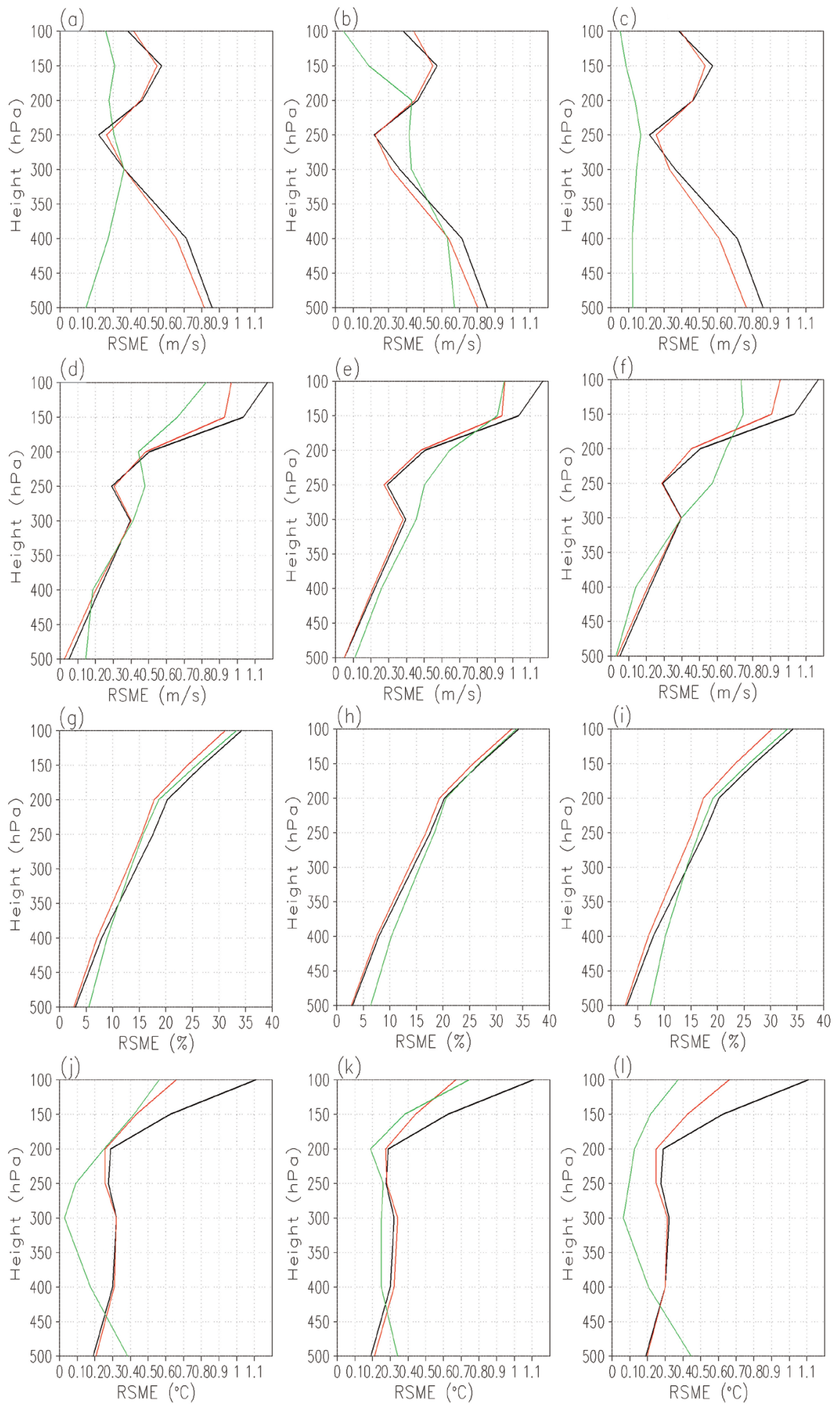

Fig. 2. Initial profiles of RSME for $(a-c)$ zonal wind, $(d-f)$ meridional wind, $(g-i)$ relative humidity, and $(j-1)$ temperature for $(a, d, g, j)$ OLD, $(b, e, h, k)$ NEW, and $(c, f, i, l)$ OLDNEW layouts, respectively for C10. Black, green and red lines represent results from analysis without "observations" assimilated, objective analysis and 3D-Var approach, respectively. 

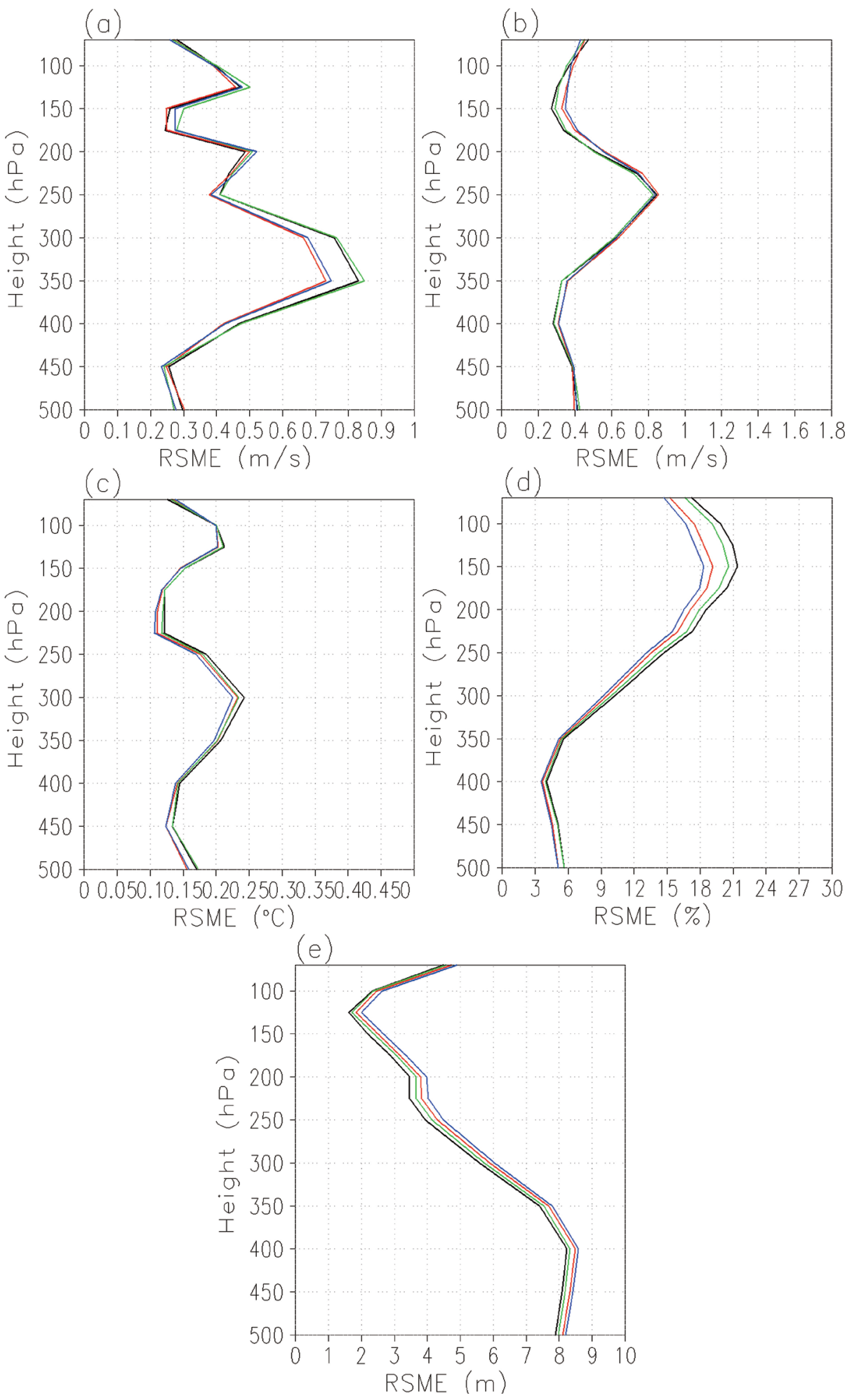

Fig. 3. Initial profiles of RSME for (a) zonal wind, (b) meridional wind, (c) temperature, (d) relative humidity, and (e) geo-potential height for C11. Black lines represent results from analysis without "observations" assimilated. Red, green and blue lines represent results respectively from the OLD, NEW, and OLDNEW layouts assimilated. 

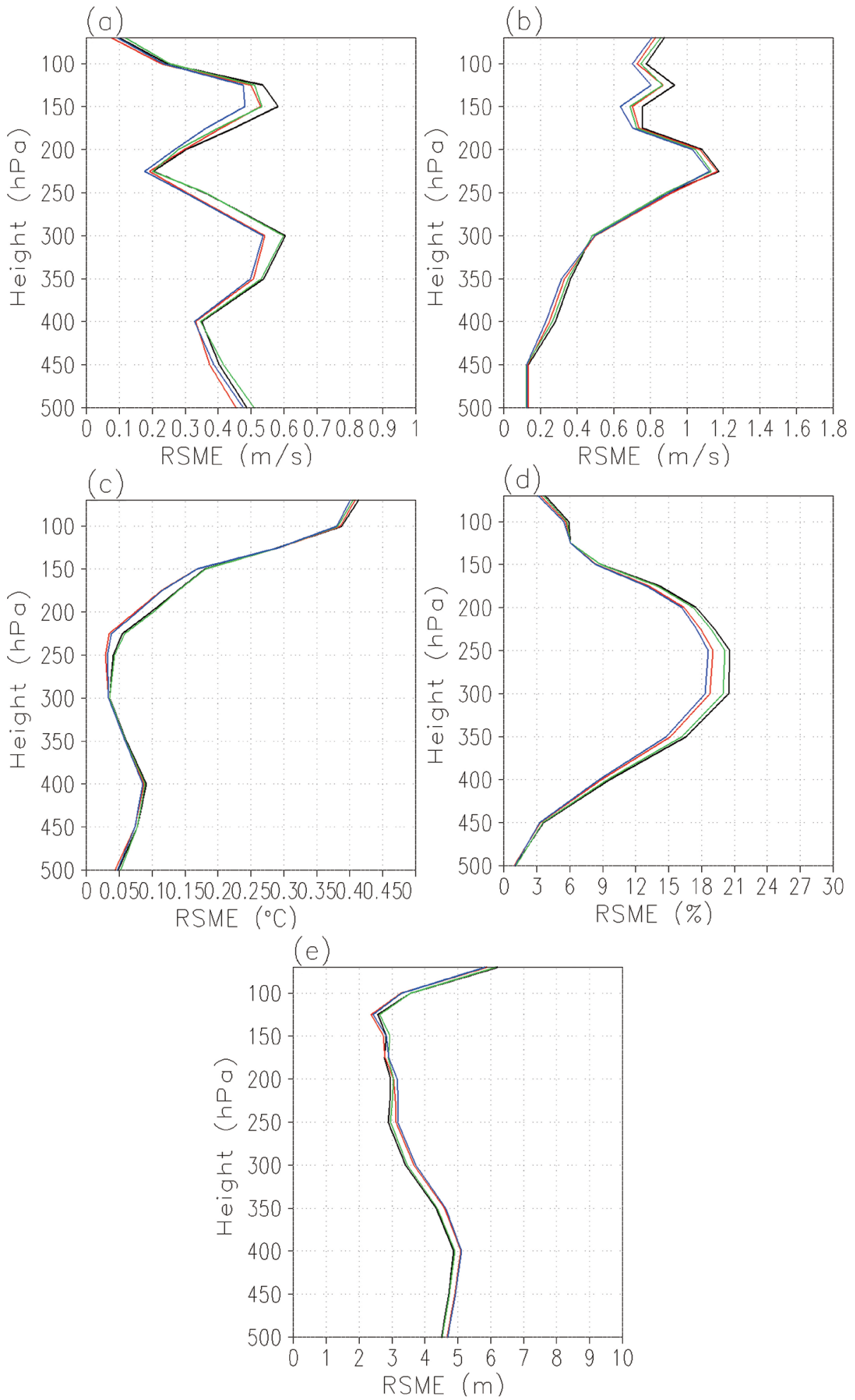

Fig. 4. Initial profiles of RSME for (a) zonal wind, (b) meridional wind, (c) temperature, (d) relative humidity, and (e) geo-potential height for C12. Black lines represent results from analysis without "observations" assimilated. Red, green and blue lines represent results respectively from the OLD, NEW, and OLDNEW layouts assimilated. 

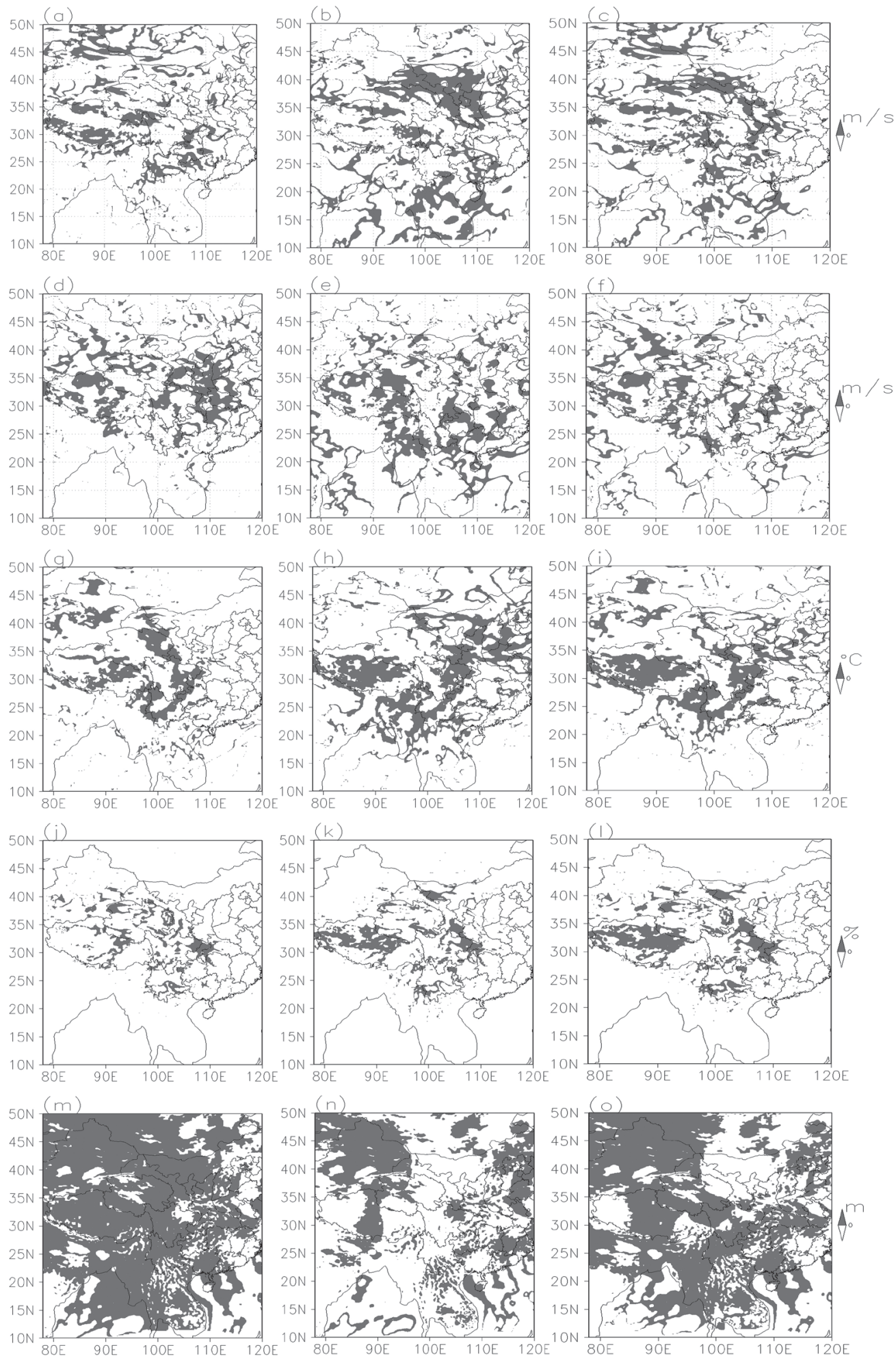

Fig. 5. Regional distribution of the RC - RE with $3 \mathrm{D}-\mathrm{VAR}$ at $500 \mathrm{hPa}$ of $\mathrm{C} 10$. (a-c) zonal wind, (d-f) meridional wind, (g-i) temperature, $(\mathrm{j}-1)$ relative humidity, and $(\mathrm{m}-\mathrm{o})$ geo-potential height for $(\mathrm{a}, \mathrm{d}, \mathrm{g}, \mathrm{j}, \mathrm{m}) \mathrm{OLD},(\mathrm{b}, \mathrm{e}, \mathrm{h}, \mathrm{k}$, n) NEW, and (c, f, i, l, o) OLDNEW layouts, respectively. 
Jiulong layout "observations" significantly improved the overall forecast results and also verified that the Jiulong has a good regional representation. The third improvement areas of the OLDNEW layout test are mainly distributed in Yunnan, Guizhou, Chongqing, and northeast Sichuan, with 12 layout points. In these areas, the improvements of zonal wind are mainly distributed near the layout site. and its surrounding area because of the poor layout points and the steep and complex terrain.

The RC - RE distribution of meridional wind (Figs. $5 \mathrm{~d}-\mathrm{f}$ ) is consistent with meridional wind, and the distribution range is relatively smaller than zonal wind.

For the RC - RE distribution of Temperature (Figs. $5 \mathrm{~g}-\mathrm{i}$ ), there are apparent differences for the forecast results in the OLD, NEW, and OLDNEW tests. The improved areas in the OLD layout test are mainly distributed in east Tibetan Plateau, east Qinghai, east Sichuan, and north and south of Yunnan. The improved areas in the NEW layout test are mainly distributed in the central and western of Tibetan Plateau, southern Qinghai, central and eastern of Sichuan and central of Yunnan. In the OLDNEW layout test forecast results, the improved areas in the OLD and NEW layout tests are combined, and are mainly distributed in three regions. The first improved areas in the OLDNEW layout test are mainly distributed in Tibet and southwest Qinghai. The second improved areas are mainly distributed in the eastern Qinghai, central and eastern of Sichuan. The third improved areas are mainly distributed in northern and central of Yunnan. The RC - RE distribution of relative humidity (Figs. $5 \mathrm{j}-1$ ) is consistent to Temperature, and not to repeat them.

For the RC - RE distribution of geo-potential height (Figs. $5 \mathrm{~m}-\mathrm{o}$ ), there are apparent differences for the forecast results in the OLD, NEW, and OLDNEW tests. The improved areas in the OLD layout test are mainly distributed in Xinjiang, Qinghai, Tibet, central and western of Sichuan, and Yunnan. The improved areas in the NEW layout test are mainly distributed in Xinjiang, Qinghai, central of Tibet, and northeast Sichuan. The improved areas in the OLDNEW layout test are mainly distributed in Xinjiang, Qinghai, central and eastern of Tibet, central and western of Sichuan, and Yunnan. In contrast to the OLD layout test, the OLDNEW and NEW layout tests are more reasonable.

In summary, the forecast results of the OLDNEW layout test are better for the zonal-meridional wind, temperature, and relative humidity, whereas the OLD and OLDNEW tests are better for the geo-potential height.

Figure 6 shows the forecast results of the OLD, NEW, and OLDNEW layout tests for $\mathrm{C} 10$ with OBJ. For zonal-meridional wind, Temperature, relative humidity, and geo-potential height, a weak difference was apparent between the OLD, NEW, and OLDNEW layout tests. The forecast results with OBS have strong regional effects. However, the 3D-VAR tends to improve the forecast results in a wide range. Overall, the $3 \mathrm{D}-\mathrm{VAR}$ is more reasonable than OBJ in the regional forecast for the OLD, NEW, and OLDNEW layout tests.

Figures 7 and 8 show the forecast results of the OLD, NEW, and OLDNEW layout tests in 2011 and 2012 with OBJ. For zonal-meridional wind, temperature, and relative humidity, the RMSEs difference is consistent with 2010. The forecast results with OBS have strong regional effects, however, the 3D-VAR tends to improve the forecast results in a wide range. Overall, the 3D-VAR is more reasonable than OBJ in the regional forecast for the OLD, NEW, and OLDNEW layout tests.

\section{Preliminary recommendations for improving the observation network over the Tibetan Plateau and its neighboring areas}

Figure 9 shows the improved areas (shaded by orange) indicated by $\mathrm{RC}-\mathrm{RE}>0$ and layout points of the OLD, NEW, and OLDNEW layout tests. The distribution of the layout points is consistent with the improved areas. The results also indicate that the "observations" of layout points have a good regional representation and geographic distribution, and therefore have a firm scientific basis. The forecast results will improve if they are coupled with the NEW layout test and the existing OLD layout test. This will also have a significant positive effect for the missing areas of OLD and NEW layout test. Therefore, it is necessary to add some new layout points in the existing OLD layout test. Based on the above analysis, we initially propose the OLDNEW layout test for the existing meteorological stations network over the Tibetan Plateau and its neighboring areas.

\section{Summary and discussion}

The OSSE was used to assess the rationality and scientific and regional representation of the existing observation system layout over the Tibetan Plateau and its neighboring areas. We drew the following preliminary conclusions:

(1) Regional distribution of the RC - RE: For 

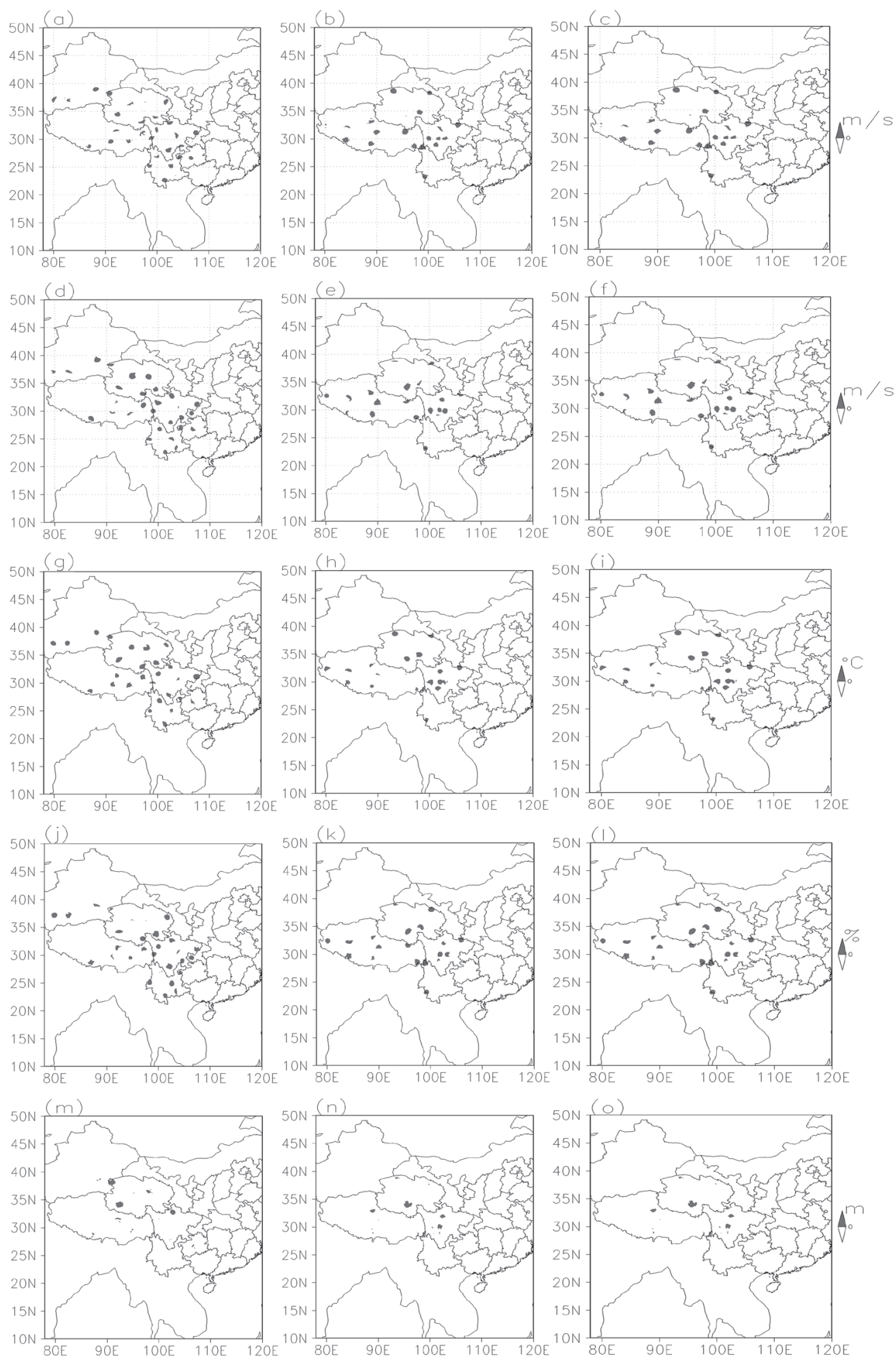

Fig. 6. Regional distribution of the $\mathrm{RC}-\mathrm{RE}$ with $\mathrm{OBJ}$ at $500 \mathrm{hPa}$ of $\mathrm{C} 10$. (a-c) zonal wind, (d-f) meridional wind, (g-i) temperature, $(\mathrm{j}-1)$ relative humidity, and $(\mathrm{m}-\mathrm{o})$ geo-potential height for $(\mathrm{a}, \mathrm{d}, \mathrm{g}, \mathrm{j}, \mathrm{m}) \mathrm{OLD},(\mathrm{b}, \mathrm{e}, \mathrm{h}, \mathrm{k}$, n) NEW, and (c, f, i, l, o) OLDNEW layouts, respectively. 

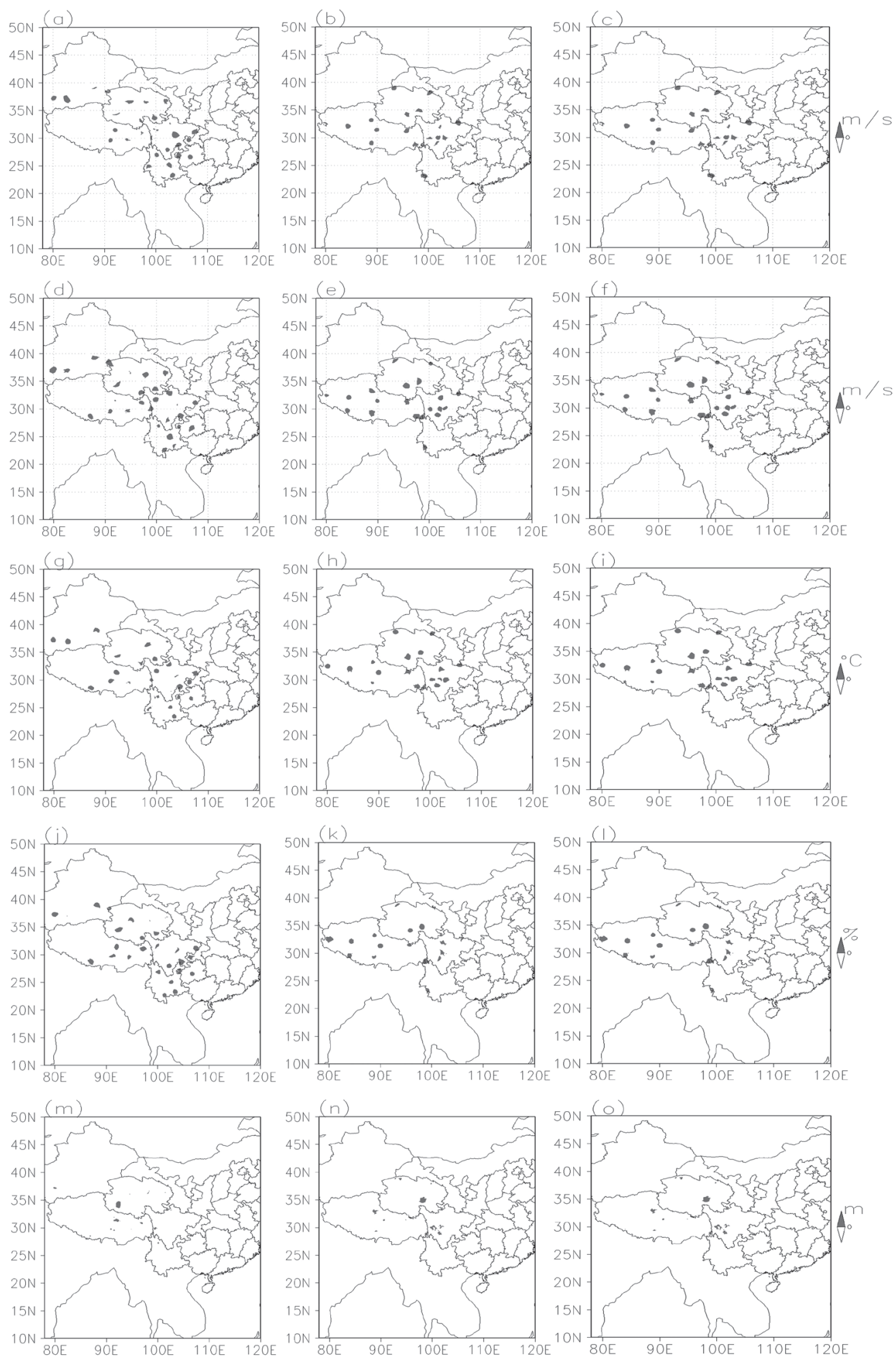

Fig. 7. Regional distribution of the RC - RE with 3D-VAR at $500 \mathrm{hPa}$ of $\mathrm{C} 11$. (a-c) zonal wind, (d-f) meridional wind, (g-i) temperature, $(\mathrm{j}-1)$ relative humidity, and $(\mathrm{m}-\mathrm{o})$ geo-potential height for $(\mathrm{a}, \mathrm{d}, \mathrm{g}, \mathrm{j}, \mathrm{m}) \mathrm{OLD},(\mathrm{b}, \mathrm{e}, \mathrm{h}, \mathrm{k}$, n) NEW, and (c, f, i, l, o) OLDNEW layouts, respectively. 

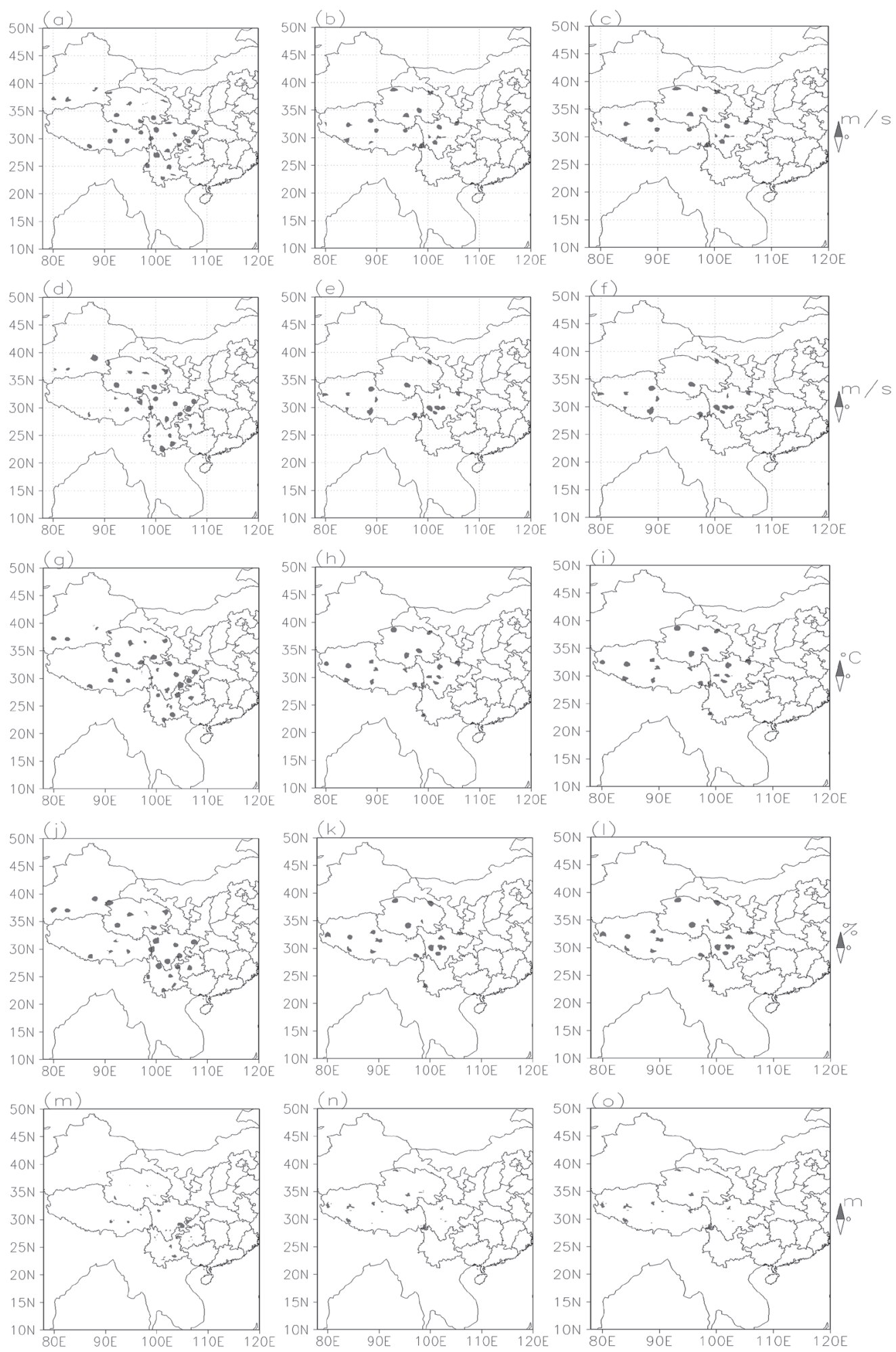

Fig. 8. Regional distribution of the RC - RE with 3D-VAR at $500 \mathrm{hPa}$ of $\mathrm{C} 12$. (a-c) zonal wind, (d-f) meridional wind, (g-i) temperature, $(j-1)$ relative humidity, and $(\mathrm{m}-\mathrm{o})$ geo-potential height for $(\mathrm{a}, \mathrm{d}, \mathrm{g}, \mathrm{j}, \mathrm{m}) \mathrm{OLD},(\mathrm{b}, \mathrm{e}, \mathrm{h}, \mathrm{k}$, n) NEW, and (c, f, i, l, o) OLDNEW layouts, respectively. 

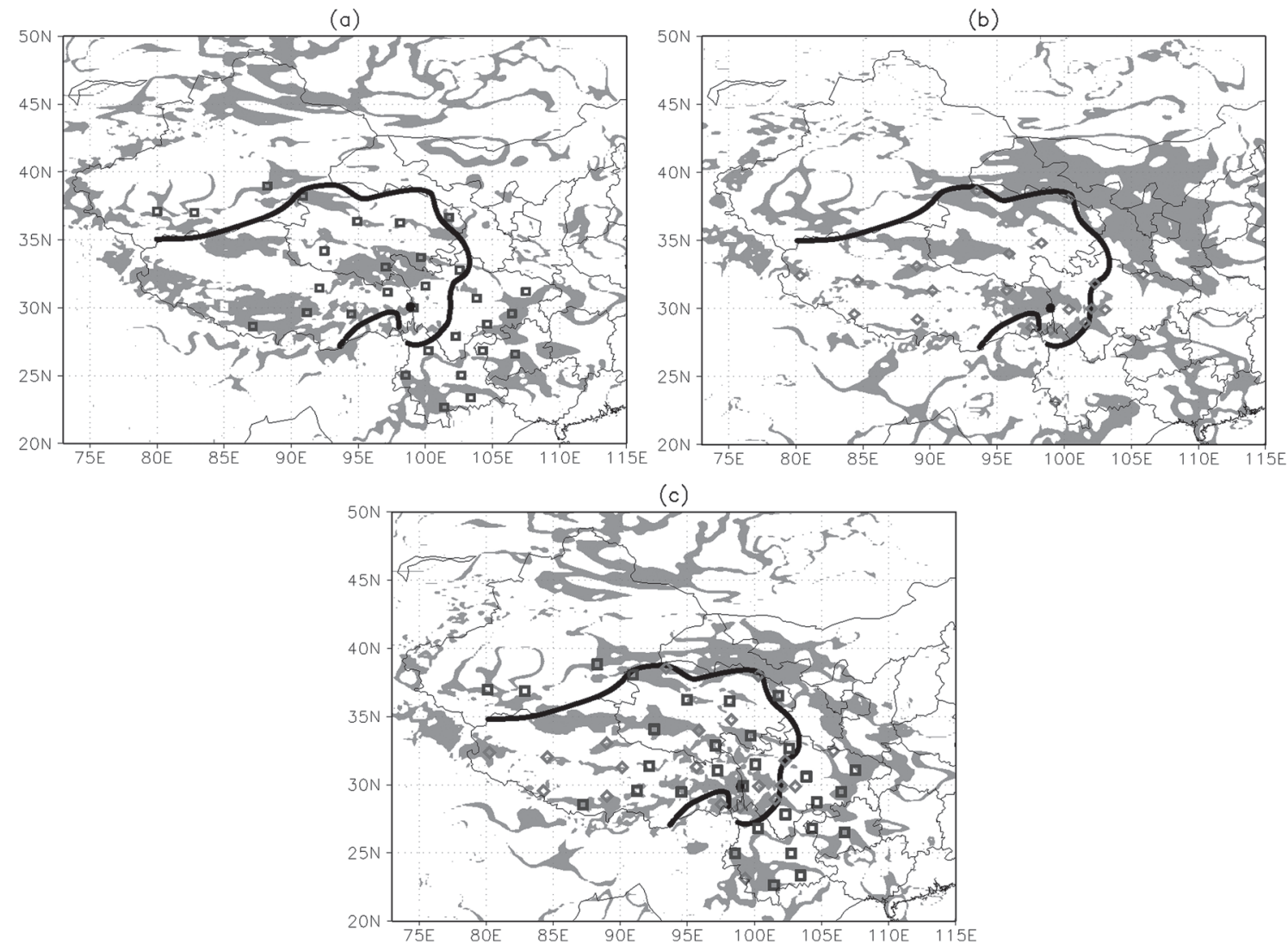

Fig. 9. The improved areas (orange-shading, $\mathrm{RC}-\mathrm{RE}>0$ ) and layout points of the (a) OLD, (b) NEW, and (c) OLDNEW layout tests.

zonal-meridional wind, temperature, and relative humidity, the forecast results of the OLDNEW layout test are closer to the EC, and $3 \mathrm{D}-\mathrm{VAR}$ is superior to OBJ. The improved areas are mainly located in the central and southern Xinjiang, Qinghai, Hexi Corridor, the main part of the Tibetan Plateau, Sichuan, central Shanxi, western Hubei, northwest and southeast of Guizhou, central and southern of Yunnan and northwest Guangxi. For geo-potential height, the forecast results of the OLD layout test are closer to the EC, and 3D-VAR is also superior to OBJ. The improved areas are mainly located in Sichuan, Qinghai, Tibet, and the Turpan Basin.

(2) Vertical distribution of the RC - RE: For zonal-meridional wind, temperature, and relative humidity, there are no significant improvements for the forecast results with the OLD, NEW, and OLDNEW layout tests, however, the RE of OLDNEW is slightly less than the OLD and NEW layout test. For geo-potential height, there is a signifi- cant improvement in the range of $400 \mathrm{hPa}-200 \mathrm{hPa}$.

(3) The OLD, NEW, and OLDNEW layout tests have a greater improvement in the Midwest Tibetan Plateau for Temperature, and OLDNEW better than OLD or NEW in term of layout, and 3D-VAR works better than OBJ in term of assimilation approach. While the OLD, NEW, and OLDNEW layout tests clearly improved in the eastern Tibetan Plateau and northwest of Sichuan for the zonal-meridional wind and relative humidity, the forecast results of OLDNEW are more consistent with EC.

The forecast results will further improve if we couple the NEW layout test with the existing OLD layout test. Such a coupling would have a significant positive effect for areas that are missing in the OLD and NEW layout test. Therefore, it is necessary to add new layout points in the existing OLD layout test. The OLDNEW layout test is the better test.

The present study is more or less kind of sensitivity study by which we tried to show how sensitive 
a planned layout of observation to the accuracy of a meso-scale modeling system from one typical case. To reach significant or robust conclusions many more cases need to be included. Moreover, other related issues such as type of data assimilations (variation, ensemble, hybrid etc.), setup of a model (resolution, physical parameterization etc.), and data homogeneity should be considered and explored in future.

\section{Acknowledgments}

This study was supported by the Special Fund for Meteorological Research in the Public Interest (GYHY201006053), the key project of the National Natural Science Foundation (No. 91337215), the National Key Basic Research Development Program Project of China (No. 2012CB417202), and Key Research and Operation Project of Southwest China Regional Meteorological Center (No.2010-1).

\section{References}

Arnold Jr., C. P., and C. H. Dey, 1986: Observing-systems simulation experiments: Past, present, and future. Bull. Amer. Meteor. Soc., 67, 687-695.

CAMS JICA Implementation Office, 2007: PRC-Japan JICA cooperation project: Advance in construction of new generation integrated meteorological monitoring network system over plateau and surrounding areas. Annual Report of CAMS, 0, 1-3.

Charney, J. G., 1949: On a physical basis for numerical prediction of large-scale motions in the atmosphere. $J$. Atmos. Sci., 6, 372-385.

Chen, S. H., J. Y. Chen, W. Y. Chang, P. L. Lin, P. H. Lin, and W. Y. Sun, 2011: Observing system simulation experiment: Development of the system and preliminary results. J. Geophys. Res., 116, D13202, doi:10.1029/2010JD015103.

Flohn, H., 1968: Contributions to meteorology of the Tibetan Highlands. Atmospheric Science Paper, $1-128$.

Liu, X. D., 1999: Influences of Qinghai-Xizang (Tibet) Plateau uplift on the atmospheric circulation, global climate and environment changes. Plateau Meteor., 18, 321-332 (in Chinese).

Liu, X. D., and Z. Y. Yin, 2002: Sensitivity of East Asian monsoon climate to the Tibetan Plateau uplift. Palaeogeogr. Palaeoclim. Palaeoecol., 22, 1075-1089 (in Chinese).

Lv, Y. Q., and Y. F. Gong, 2006: Atmospheric heat source/ sink change characteristics over Qinghai-Xizang Plateau and Its vicinity region in summer of 2001 and 2003. Plateau Meteor., 25, 195-202 (in Chinese).

Marseille, G. J., and A. Stoffelen, 2003: Simulation of wind profiles from a space-borne Doppler wind lidar. Quart. J. Roy. Meteor. Soc., 129, 3079-3098.
Masutani, M., J. S. Woollen, S. J. Lord, T. J. Kleespies, G. D. Emmitt, H. Sun, S. A. Wood, S. Greco, J. Terry, R. Treadon, and K. A. Campana, 2006: Observing system simulation experiments at NCEP. NCEP Office Note, 451.

Masutani, M., J. S. Woollen, S. J. Lord, G. D. Emmitt, T. J. Kleespies, S. A. Wood, S. Greco, H. Sun, J. Terry, V. Kapoor, R. Treadon, and K. A. Campana, 2010: Observing system simulation experiments at the national centers for environmental prediction. J. Geophys. Res., 115, D07101, doi:10.1029/2009JD012528.

Shen, G. X., and G. B. He, 2008: The observed facts analysis of southwest vortex from 2000 to 2007. Plateau Mt. Meteor. Res., 28, 59-65 (in Chinese).

Staff members, 1984: Collections of the Qinghai-Xizang (Tibet) Plateau Meteorological Experiment (Part one). Science Press, Beijing, 1-343 (in Chinese).

Staff members, 1984: Collections of the Qinghai-Xizang (Tibet) Plateau Meteorological Experiment (Part two). Science Press, Beijing, 1-280 (in Chinese).

Staff members, 1987: Collections of the Qinghai-Xizang (Tibet) Plateau Meteorological Experiment (Part three). Science Press, Beijing, 1-215 (in Chinese).

Tao, S. Y., L. S. Chen, X. D. Xu, and Y. S. Xiao, 1998: Progress in the second Tibetan Plateau Atmospheric Experiment (TIPEX) (Part one). China Meteorological Press, Beijing, 1-348 (in Chinese).

Tao, S. Y., L. S. Chen, X. D. Xu, and Y. S. Xiao, 1998: Progress in the second Tibetan Plateau Atmospheric Experiment (TIPEX) (Part two). China Meteorological Press, Beijing, 1-396 (in Chinese).

Tao, S. Y., L. S. Chen, X. D. Xu, and Y. S. Xiao, 1998: Progress in the Second Tibetan Plateau Atmospheric Experiment (TIPEX) (Part three). China Meteorological Press, Beijing, 1-203 (in Chinese).

Wang, B., and I. Oranski, 1987: Study of a heavy rain vortex formed over the eastern flank of the Tibetan Plateau. Mon. Wea. Rev., 115, 1370-1393.

Wang, Q., P. W. Guo, H. W. Zhou, F. Y. Zhang, and Z. G. Zhang, 2011: Climatic character of heat sources in Tibetan Plateau. J. Meteor. Sci., 31, 179-186 (in Chinese).

Wang, X., Y. Q. Li, S. H. Yu, and X. W. Jiang, 2009: Statistical study on the Plateau low vortex activities, Plateau Meteor, 28, 64-71 (in Chinese).

Wu, G. X., J. Y. Mao, A. M. Duan, and Q. Zhang, 2004: Recent progress in the study on the impacts of Tibetan Plateau on Asian summer climate. Acta Meteor. Sinica, 62, 528-540 (in Chinese).

Wu, G. X., Y. J. Zheng, and Y. M. Liu, 2013: Dynamical and thermal problems in vortex development and movement. Part II: Generalized slantwise vorticity development. Acta Meteor. Sinica, 27, 15-25 (in Chinese).

Xu, X. D., S. Y. Tao, J. Z. Wang, L. S. Chen, L. Zhou, and X. R. Wang, 2002: The relationship between water 
vapor transport features of Tibetan Plateau - monsoon "large triangle" affecting regions and drought-flood abnormality of China. Acta Meteor. Sinica, 60, 257-266 (in Chinese).

Xu, X. D., Q. J. Miao, J. Z. Wang, and X. J. Zhang, 2003: The water vapor transport model at the regional boundary during meiyu period. Adv. Atmos. Sci., 20, 333-342.

Yanai, M., C. Li, and Z. Song, 1992: Seasonal heating of the Tibetan Plateau and its effects on the evolution of the Asian summer monsoon. J. Meteor. Soc. Japan, 70, 319-351.

Yanai, M., and C. F. Li, 1994: Mechanism of heating and the boundary layer over the Tibetan Plateau. Mon. Wea. Rev., 122, 305-323.

Yang, K., X. F. Guo, and B. Y. Wu, 2010: Recent trends in surface sensible heat flux on the Tibetan Plateau. Sci. China Earth Sci., 54, 19-28.
Ye, D. Z., and Y. X. Gao, 1979: The Plateau Meteorology. The Science Press, Beijing, 1-278 (in Chinese).

Yu, S. H., 2002: Water vapor imagery of vortex moving process over Qinghai-Xizang Plateau. Plateau Meteor., 21, 199-204 (in Chinese).

Yu, S. Q., and J. Z. Wang, 2005: Advance in Sino-Japan Joint Research Center of Meteorological Disaster (JICA Project). Annual Report of CAMS, 0, 1-2 (in Chinese).

Zheng, Y. J., G. X. Wu, and Y. M. Liu, 2013: Dynamical and thermal problems in vortex development and movement. Part I: A PV-Q View. Acta Meteor. Sinica, 71, 185-197 (in Chinese).

Zhong, S. S., J. H. He, Z. Y. Guan, and M. Wen, 2009: Climatic characteristics of the atmospheric heat source over the Tibetan Plateau during 1961-2001. Acta Meteor. Sinica, 67, 407-416 (in Chinese). 